WU B 03-05

hep-ph/0309071

September 2003

\title{
Signatures of the handbag mechanism in wide-angle photoproduction of pseudoscalar mesons
}

\author{
H.W. Huang \\ Department of Physics, University of Colorado, Boulder, CO 80309-0390, USA \\ R. Jakob and P. Kroll \\ Fachbereich Physik, Universität Wuppertal, D-42097 Wuppertal, Germany \\ K. Passek-Kumerički \\ Theoretical Physics Division, Rudjer Bošković Institute, HR-10002 Zagreb, Croatia
}

\begin{abstract}
Wide-angle photoproduction of pseudoscalar mesons is investigated under the assumption of dominance of the handbag mechanism considering both quark helicity flip and non-flip. The partonic subprocess, meson photoproduction off quarks, is analysed with the help of a covariant decomposition of the subprocess amplitudes which is independent of a specific meson generation mechanism. As examples of subprocess dynamics, however, the twist-2 as well as two-particle twist-3 contributions are explicitly calculated. Characteristic features of the handbag approach are discussed in dependence upon the relative magnitudes of the invariant functions. Differential cross sections and spin correlations are predicted to show a characteristic behaviour which allows to test the underlying assumption of handbag dominance.
\end{abstract}




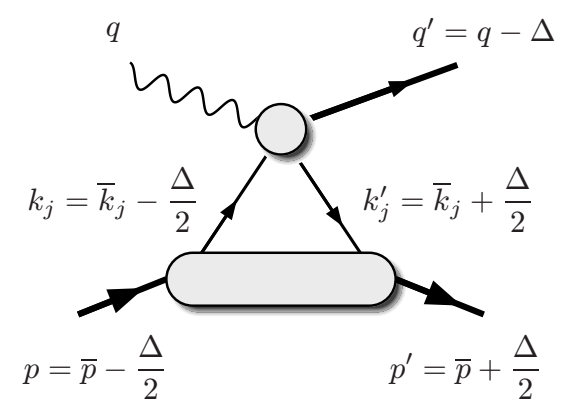

Figure 1: The handbag diagram for photo- and electroproduction of mesons. The large blob represents a baryon GPD, while the small one stands for meson photo- and electroproduction off partons. The momenta of the various particles are indicated.

\section{Introduction}

Recently, the importance of the handbag mechanism in hard exclusive reactions has been realised. The handbag mechanism is characterised by the fact that only one quark from the incoming and one from the outgoing nucleon participate in the hard subprocess, while all other partons are spectators. Factorisation properties have been shown to hold for Compton scattering and for meson electroproduction in the both kinematical regions: the deep virtual region characterised by a large virtuality $Q^{2}$ of the incoming photon and a small squared invariant momentum transfer $t$ from the incoming to the outgoing proton, and the wide-angle region where $-t$ (and $-u$ ) are regarded as the large scale, while $Q^{2}$ is less than $-t$. It is to be emphasised that for deep virtual processes, all order proofs of factorisation exist, while for the wide-angle region factorisation has only been shown to hold to next-to-leading order in Compton scattering and to leading order in photo- and electroproduction of mesons as yet.

As illustrated in Fig. 1, the amplitudes for hard exclusive processes factorise into a partonlevel subprocess, e.g., meson photo- or electroproduction off quarks, $\gamma^{(*)} q \rightarrow M q$, and generalised parton distributions (GPDs) [1] describing the soft hadron-parton transitions. Factorisation is particularly simple in the wide-angle region. Instead of convolution as occurring in deep virtual processes, the wide-angle amplitudes appear as products of subprocess amplitudes and $t$-dependent form factors which represent $1 / x$-moments of GPDs. There is another difference between deep virtual and wide-angle electroproduction of mesons. The deep virtual process [2] is dominated by contributions from longitudinally polarised virtual photons for $Q^{2} \rightarrow \infty$; those from transversally polarised photons are suppressed by $1 / Q^{2}$. For these amplitudes, factorisation breaks down [3]. For wide-angle electroproduction, both photon polarisations contribute to the same twist order, there is no break-down of factorisation [4]; the limit $Q^{2} \rightarrow 0$ is therefore unproblematic.

Whereas for Compton scattering reasonable agreement with experiment has been found despite the rather low values of $Q^{2}$ or $-t,-u$ at which data is available, in meson electroproduction the magnitude of the cross sections calculated within the handbag approach turns out to fail in describing the data $[4,5]$. The reason for this defect is presumably the onegluon exchange mechanism for the generation of the meson (see Fig. 2) and not the handbag mechanism itself. Although the one-gluon exchange mechanism combined with the leadingtwist meson distribution amplitude dominates for asymptotically large scales, it may fail in the kinematical situation accessible to current experiments which is characterised by scales 

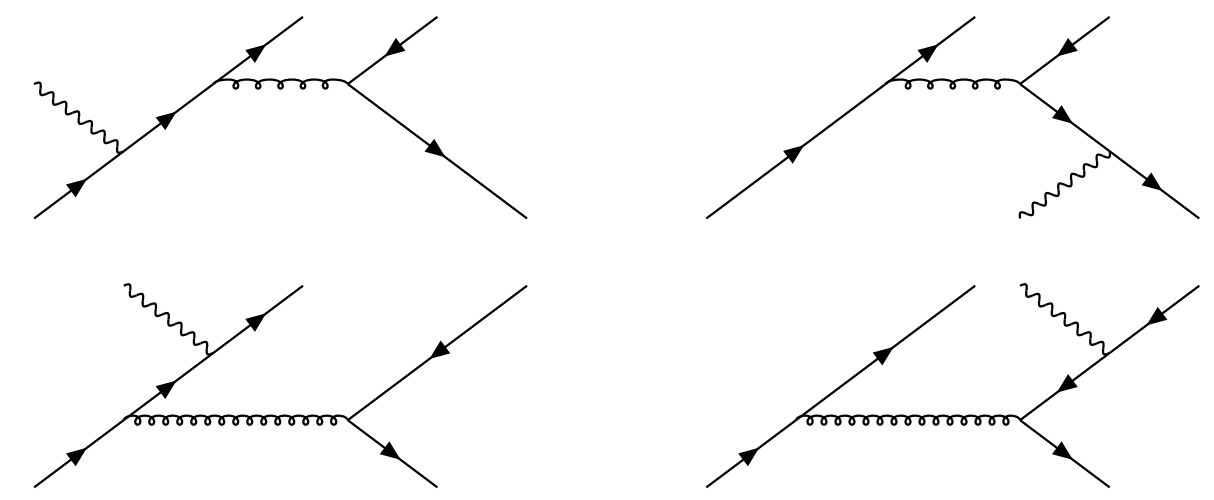

Figure 2: Lowest order Feynman graphs contributing to the subprocess $\gamma^{(*)} q \rightarrow M q$ to leading twist. The upper quark and antiquark lines enter the meson's wave function. The internal curly lines represent hard gluons.

of a few $\mathrm{GeV}^{2}$ only. The lowest-order Feynman graphs contributing to $\gamma^{(*)} q \rightarrow M q$ are the same as those occurring in the leading-twist calculations of the meson form factor [6]. As is well-known (see, for instance [7,8]), the leading-twist results for the pion form factor are by a factor 3 to 4 below the admittedly poor data available at present [9]. In semi-exclusive pion photoproduction [10] which bases on the same subprocess $\gamma^{(*)} q \rightarrow M q$ too, the normalisation also fails in comparison with experiment. In the light of these results, a failure by order of magnitude for the photo- and electroproduction cross sections is to be anticipated. Thus, it is probably insufficient to consider the one-gluon exchange mechanism for which only zero quark-antiquark spatial separations are taken into account and the quark transverse momentum is neglected. It entails a factor $f_{M}^{2} / s$ in the photo- and electroproduction cross section, which is indeed a tiny number at large $s$ ( $f_{M}$ is the meson's decay constant).

How can this situation be remedied? Suppose handbag factorisation continues to hold. Then one may think, for instance, of higher-twist or power corrections to the meson generation, resummation of perturbative corrections or long-distance meson wave function effects, which may enhance the leading-twist amplitude decisively. For instance, by insertion of an infinite number of fermionic loops in the hard gluon propagator and interpreting the ambiguities in the resummation of these loop effects (known as infrared renormalons) as a model of higher-twist contributions an enhancement of a factor 3 to 4 is estimated [11].

In a more pessimistic scenario, one would consider substantial non-factorising contributions to photo- and electroproduction of mesons at moderately large values of the hard scale. The purpose of this work is to investigate the first scenario, i.e., assuming the validity of the handbag mechanism for photoproduction of pseudoscalar mesons, but allowing for more general subprocess amplitudes than the one-gluon exchange ones. The generalisation of our investigation to electroproduction in the wide-angle and the deep virtual region is straightforward.

The plan of the paper is the following: In Sect. 2 we recapitulate the handbag mechanism in photoproduction of pseudoscalar mesons $(P)$, extend it in order to include more general mechanisms for the meson generation and present some kinematical details. The subprocess $\gamma q \rightarrow P q$ is discussed in terms of a covariant decomposition in Sect. 3, and the twist-2 and twist-3 contributions to the subprocess are calculated. Sect. 4 is devoted to the discussion of 
characteristic predictions for meson photoproduction from the handbag approach which may hold even if the normalisation of the cross section is not yet understood. The paper ends with a summary.

\section{The handbag mechanism}

The handbag mechanism for wide-angle scattering reactions was first developed for Compton scattering $[12,13]$ and subsequently applied to photo- and electroproduction of mesons [4]. In this paper we will restrict ourselves to photoproduction of pseudoscalar mesons, $\gamma B_{1} \rightarrow P B_{2}$, where $B_{i}$ denotes a member of the lowest-lying baryon octet. For the sake of legibility, we consider here the case of charged and uncharged pions, and, hence, of nucleons, leaving the generalisations to other pseudoscalar mesons to the end of the paper. A prerequisite to the application of the handbag mechanism are Mandelstam variables, $s,-t,-u$, that are large as compared to $\Lambda^{2}$, where $\Lambda$ is a hadronic scale of order $1 \mathrm{GeV}$. It is of advantage to work in a symmetrical frame which is a c.m.s. rotated in such a way that the momenta of the incoming $(p)$ and outgoing $\left(p^{\prime}\right)$ baryons have the same light-cone plus components:

$$
p=\left[p^{+}, \frac{m^{2}-t / 4}{2 p^{+}},-\frac{1}{2} \boldsymbol{\Delta}_{\perp}\right], \quad p^{\prime}=\left[p^{+}, \frac{m^{2}-t / 4}{2 p^{+}}, \frac{1}{2} \boldsymbol{\Delta}_{\perp}\right],
$$

where $m$ is the mass of the nucleon, for the definition of other momenta see Fig. 1 . The chief advantage of the symmetric frame is that the skewness

$$
\xi=\frac{\left(p-p^{\prime}\right)^{+}}{\left(p+p^{\prime}\right)^{+}}
$$

vanishes. The baryonic blob in the handbag, see Fig. 1, is viewed as a sum over all possible parton configurations as in deep inelastic lepton-nucleon scattering (DIS). The crucial assumptions in the handbag approach are that of restricted parton virtualities $k_{i}^{2}<\Lambda^{2}$, and of intrinsic transverse parton momenta $\mathbf{k}_{\perp \mathbf{i}}$, defined with respect to their parent hadron's momentum, which satisfy $k_{\perp i}^{2} / x_{i}<\Lambda^{2}$, where $x_{i}$ is the momentum fraction that the parton $i$ carries.

One can then show $[4,13]$ that the subprocess Mandelstam variables $\hat{s}$ and $\hat{u}$ are the same as the ones for the full process, photoproduction off baryons, up to corrections of order $\Lambda^{2} / t$ $(\hat{t}=t)$ :

$$
\hat{s}=\left(k_{j}+q\right)^{2} \simeq(p+q)^{2}=s, \quad \hat{u}=\left(k_{j}-q^{\prime}\right)^{2} \simeq\left(p-q^{\prime}\right)^{2}=u,
$$

where $k_{j}$ denotes the momentum of the active parton, i.e., the one to which the photon couples. The active partons are approximately on-shell, move collinear with their parent hadrons and carry a momentum fraction close to unity, $x_{j}, x_{j}^{\prime} \simeq 1$. Thus, like in deep virtual exclusive scattering, the physical situation is that of a hard parton-level subprocess $\gamma q_{a} \rightarrow P q_{b}$ and a soft emission and reabsorption of quarks from the baryon. The light-cone helicity amplitudes [14] for wide-angle photoproduction then read

$$
\begin{aligned}
& \mathcal{M}_{0+, \mu+}\left(\gamma B_{1} \rightarrow P B_{2}\right) \\
& =\frac{e}{2} \sum_{a, b=u, d, s}\left[\mathcal{H}_{0+, \mu+}^{P(a b)}(s, t)\left(R_{V, B_{1} \rightarrow B_{2}}^{a b}(t)+R_{A, B_{1} \rightarrow B_{2}}^{a b}(t)\right)\right. \\
& \left.\quad+\mathcal{H}_{0-, \mu-}^{P(a b)}(s, t)\left(R_{V, B_{1} \rightarrow B_{2}}^{a b}(t)-R_{A, B_{1} \rightarrow B_{2}}^{a b}(t)\right)\right]
\end{aligned}
$$




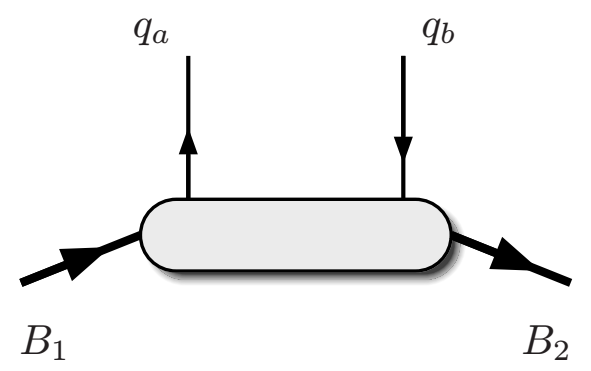

Figure 3: The GPD and the respective form factors $R_{i, B_{1} \rightarrow B_{2}}^{a b}$ for $B_{1} \rightarrow B_{2}$ transitions due to emission of a quark with flavour $a$ and reabsorption of a quark with flavour $b$.

and

$$
\begin{aligned}
& \mathcal{M}_{0-, \mu+}\left(\gamma B_{1} \rightarrow P B_{2}\right) \\
& =\frac{e}{2} \frac{\sqrt{-t}}{2 m} \sum_{a, b=u, d, s}\left[\mathcal{H}_{0+, \mu+}^{P(a b)}(s, t)+\mathcal{H}_{0-, \mu-}^{P(a b)}(s, t)\right] R_{T, B_{1} \rightarrow B_{2}}^{a b}(t),
\end{aligned}
$$

where $\mu$ denotes the helicity of the photon and $e$ is the positron charge. The frame has been chosen such that the momentum transfer $\Delta^{\mu}$ has a positive 1-component and a zero 2-component. The helicities of the baryons in $\mathcal{M}$ and of the quarks in the hard scattering amplitude $\mathcal{H}$ are labelled by their signs only for the sake of legibility. The amplitudes for other helicity configurations follow from parity invariance:

$$
\mathcal{M}_{0 \nu^{\prime}, \mu \nu}^{P}=(-1)^{\nu-\nu^{\prime}} \mathcal{M}_{0-\nu^{\prime},-\mu-\nu}^{P} .
$$

An analogous relation holds for the parton-level amplitudes $\mathcal{H}$.

The hard scattering amplitudes can be computed from the leading-order Feynman graphs shown in Fig. 2 and the twist-2 meson distribution amplitude. As a consequence of this leading-twist generation of the meson and the masslessness of the quarks there is no quark helicity flip. A more general mechanism for the generation of the meson, which we discuss in the next section, may allow quark helicity flips. The form factors $R_{i, B_{1} \rightarrow B_{2}}^{a b}$ represent $1 / x$ moments of GPDs at zero skewness, where $x=\left(k_{j}+k_{j}^{\prime}\right)^{+} /\left(p+p^{\prime}\right)^{+}$is the average momentum fraction the two quarks carry. The form factors parameterise the soft physics that controls the emission of a quark with flavour $a$ and the reabsorption of a quark with flavour $b$ by a baryon (see Fig. 3). The flavours of the emitted and reabsorbed quarks fix the quantum numbers of the final baryon uniquely for given initial baryon. The representation (4), (5), which requires the dominance of the plus components of the baryon matrix elements, is a nontrivial feature given that, in contrast to DIS and deep virtual exclusive reactions, not only the plus components of the nucleon momenta, but also their minus and transverse components are large in this case [13].

For the proton-proton transitions we use the simplifying notation $(i=V, A, T)$

$$
R_{i, p \rightarrow p}^{a a}(t) \equiv R_{i}^{a}(t) .
$$

These form factors are related to the zero skewness proton GPDs [1] by

$$
R_{V}^{a}(t)=\int_{-1}^{1} \frac{d x}{x} \operatorname{sign}(x) H^{a}(x, 0 ; t),
$$




$$
\begin{aligned}
& R_{A}^{a}(t)=\int_{-1}^{1} \frac{d x}{x} \widetilde{H}^{a}(x, 0 ; t), \\
& R_{T}^{a}(t)=\int_{-1}^{1} \frac{d x}{x} \operatorname{sign}(x) E^{a}(x, 0 ; t),
\end{aligned}
$$

where we have used Ji's notation for the GPDs. Note that $x$ runs from -1 to +1 . As usual, a parton with a negative momentum fraction is reinterpreted as an antiparton with a positive momentum fraction. The GPD $\widetilde{E}^{a}$ and its associated form factor decouples in the symmetric frame. The form factor $R_{T}^{a}$, which was ignored in the previous work on wideangle photoproduction [4], is suppressed by at least $\Lambda / \sqrt{-t}$ as compared to the other two form factors. The form factors $R_{i}^{a}$ for the production of pseudoscalar mesons are similar but not identical to those appearing in wide-angle Compton scattering $[13,15]$; for instance, as a consequence of charge conjugation symmetry, active unpolarised quarks and antiquarks contribute with opposite sign in photoproduction of pseudoscalar mesons but with the same sign in Compton scattering and photoproduction of vector mesons. The GPDs, describing the properties of the proton, are the same in both processes, as follows from the universality property of the GPDs [1].

The form factors for other baryon transitions can be related to the $p \rightarrow p$ ones by flavour symmetry. Thus, as a consequence of isospin invariance, one has $(i=V, A, T)$

$$
R_{i, n \rightarrow n}^{d d}=R_{i}^{u} ; \quad R_{i, n \rightarrow n}^{u u}=R_{i}^{d} ; \quad R_{i, n \rightarrow n}^{s s}=R_{i}^{s} .
$$

For photoproduction of charged pions, flavour non-diagonal GPDs occur. These GPDs and, hence, the associated form factors are related to the diagonal $p \rightarrow p$ ones by [16]

$$
R_{i, p \rightarrow n}^{u d} \equiv R_{i}^{\pi^{+}}=R_{i, n \rightarrow p}^{d u} \equiv R_{i}^{\pi^{-}}=R_{i}^{u}-R_{i}^{d} .
$$

As we see, only the isovector combination contributes to $\pi^{ \pm}$photoproduction. The axial form factor $F_{A}$ [13] and $R_{A}$ for $\pi^{ \pm}$photoproduction are both related to the isovector combination of $\widetilde{H}$. Owing to the different moments that the form factors represent, in addition to the valence quarks, $u$ and $d$ sea quarks contribute to $F_{A}$, whereas they cancel in $R_{A}$.

The photoproduction amplitudes for quark helicity flip can be derived in a way fully analogous to the derivation of the amplitudes (4) [4]. The result is

$$
\begin{aligned}
\mathcal{M}_{0+, \mu+}^{T}\left(\gamma B_{1} \rightarrow P B_{2}\right)= & -e \frac{\sqrt{-t}}{2 m} \sum_{a, b=u, d, s}\left[\mathcal{H}_{0-, \mu+}^{P(a b)}(s, t)-\mathcal{H}_{0+, \mu-}^{P(a b)}(s, t)\right] \\
& \times\left[S_{S, B_{1} \rightarrow B_{2}}^{a b}(t)+\frac{1}{2} S_{V, B_{1} \rightarrow B_{2}}^{a b}(t)\right] \\
\mathcal{M}_{0-, \mu+}^{T}\left(\gamma B_{1} \rightarrow P B_{2}\right)= & e \sum_{a, b=u, d, s}\left[\mathcal{H}_{0-, \mu+}^{P(a b)}(s, t) S_{T, B_{1} \rightarrow B_{2}}^{a b}(t)\right. \\
& \left.-\frac{t}{4 m^{2}}\left[\mathcal{H}_{0-, \mu+}^{P(a b)}(s, t)-\mathcal{H}_{0+, \mu-}^{P(a b)}(s, t)\right] S_{S, B_{1} \rightarrow B_{2}}^{a b}(t)\right] .
\end{aligned}
$$

These amplitudes are to be added to those given in (4). Again we denote the form factors for $p \rightarrow p$ transitions by $S_{i}^{a}$. Analogously to the form factors $R_{i}^{a}$, they represent $1 / x$ moments of quark helicity flip distributions

$$
S_{T}^{a}=\int_{-1}^{1} \frac{d x}{x} \operatorname{sign}(x) H_{T}^{a}(x, 0 ; t)
$$




$$
\begin{aligned}
S_{S}^{a} & =\int_{-1}^{1} \frac{d x}{x} \operatorname{sign}(x) \widetilde{H}_{T}^{a}(x, 0 ; t), \\
S_{V}^{a} & =\int_{-1}^{1} \frac{d x}{x} \operatorname{sign}(x) E_{T}^{a}(x, 0 ; t),
\end{aligned}
$$

for $i=T, S, V$. The signum functions which control the relative signs between quark and antiquark contributions, manifest the charge-conjugation properties of helicity-flip distributions. The form factors for $B_{1} \rightarrow B_{2}$ transitions can be related to the $p \rightarrow p$ ones in the same fashion as for $R_{i}$, see (10). The new proton GPDs are defined in [14] (see also [17]):

$$
\begin{aligned}
& \frac{1}{2} \int \frac{d z^{-}}{2 \pi} \mathrm{e}^{i x \bar{p}^{+} z^{-}}\left\langle p^{\prime}, \lambda^{\prime}\left|\bar{\Psi}_{a}(-\bar{z} / 2) i \sigma^{+i} \Psi_{a}(\bar{z} / 2)\right| p, \lambda\right\rangle \\
& =\frac{1}{\bar{p}^{+}} \bar{u}\left(p^{\prime}, \lambda^{\prime}\right)\left[H_{T}^{a}(x, \xi ; t) i \sigma^{+i}+\widetilde{H}_{T}^{a}(x, \xi ; t) \frac{\bar{p}^{+} \Delta^{i}-\Delta^{+} \bar{p}^{i}}{m^{2}}\right. \\
& \left.\quad+E_{T}^{a}(x, \xi ; t) \frac{\gamma^{+} \Delta^{i}-\Delta^{+} \gamma^{i}}{2 m}+\widetilde{E}_{T}^{a} \frac{\gamma^{+} \bar{p}^{i}-\bar{p}^{+} \gamma^{i}}{m}\right] u(p, \lambda),
\end{aligned}
$$

where $i=1,2$ is a transverse index,

$$
\bar{p}=\frac{1}{2}\left(p+p^{\prime}\right),
$$

and $\bar{z}=\left[0, z^{-}, \mathbf{0}_{\perp}\right]$. At $\xi=0$ the GPD $\widetilde{E}_{T}^{a}$ vanishes. As can be seen from the helicity configurations of the involved quarks and baryons [14], in contrast to $H_{T}^{a}$ the GPDs $E_{T}^{a}$ and $\widetilde{H}_{T}^{a}$ involve components of the baryon wave functions where the parton helicities do not add up to the helicities of the baryons. In other words, parton configurations with nonzero orbital angular momentum contribute to it. As inspection of the parton-hadron helicity matrix elements [14] reveals, these GPDs as well as the corresponding form factors $S_{V}^{a}$ and $S_{S}^{a}$ are expected to be suppressed as compared to $S_{T}^{a}$ by at least $1 / \sqrt{-t}$ and $1 / t$, respectively. In this sense the situation is analogous to the expected suppression of the Pauli form factor with respect to the Dirac one, which should be at least $\propto 1 / \sqrt{-t}$. On the other hand, there is no argument why $S_{T}^{a}$ could be suppressed as compared to $R_{V}^{a}$. Thus, quark helicity flip is suppressed by the subprocess amplitudes only. It is worth mentioning that all GPDs, those for quark helicity flip as well as those for non-flip, are real valued, as a consequence of time reversal invariance.

\section{The parton-level subprocess $\gamma q_{a} \rightarrow P q_{b}$}

\subsection{A covariant decomposition}

The helicity amplitudes for pseudoscalar meson photoproduction off quarks, $\gamma q_{a} \rightarrow P q_{b}$, can be decomposed covariantly as

$$
\mathcal{H}_{0 \lambda^{\prime}, \mu \lambda}^{P(a b)}(\hat{s}, \hat{t})=\sum_{i=1}^{4} C_{i}^{P(a b)}(\hat{s}, \hat{t}) \bar{u}\left(k^{\prime}, \lambda^{\prime}\right) Q_{i}(\mu) u(k, \lambda),
$$

where $\hat{s}, \hat{u}$, and $\hat{t}$ denote the subprocess Mandelstam variables. A suitable set of covariants was given by Chew et al. (CGLN) [18]:

$$
Q_{1}=2 \gamma_{5}\left[q^{\prime} \cdot q \bar{k} \cdot \varepsilon-q^{\prime} \cdot \varepsilon \bar{k} \cdot q\right]
$$




$$
\begin{aligned}
Q_{2} & =2 \gamma_{5}[\bar{k} \cdot q \notin-\bar{k} \cdot \varepsilon \not], \\
Q_{3} & =\gamma_{5}\left[q^{\prime} \cdot q \notin-q^{\prime} \cdot \varepsilon \not q\right], \\
Q_{4} & =\gamma_{5} \notin \not q,
\end{aligned}
$$

where $\varepsilon$ is the polarisation vector of the photon. The covariants $Q_{i}$ are manifestly gauge invariant and encode the helicity structure of the process, whereas the invariant functions $C_{i}^{P(a b)}$ depend on the dynamics. In the symmetric frame, the various momenta and the photon's polarisation vector have the following representation:

$$
\begin{array}{rlrl}
\bar{k} & =\left[k^{+}, k^{-}, \mathbf{0}_{\perp}\right], & \Delta & =\left[0,0, \sqrt{-\hat{t}} \mathbf{e}_{1}\right], \\
q & =\left[k^{-}, k^{+}, \frac{1}{2} \sqrt{-\hat{t}} \mathbf{e}_{1}\right], & q^{\prime} & =\left[k^{-}, k^{+},-\frac{1}{2} \sqrt{-\hat{t}} \mathbf{e}_{1}\right], \\
\varepsilon(\mu) & =\left[\frac{\mu}{2} \sqrt{\frac{-\hat{t}}{\hat{s}}},-\frac{\mu}{2} \sqrt{\frac{-\hat{t}}{\hat{s}}}, \frac{\mu}{\sqrt{2}} \sqrt{\frac{-\hat{u}}{\hat{s}}} \mathbf{e}_{1}-\frac{i}{\sqrt{2}} \mathbf{e}_{2}\right],
\end{array}
$$

where the plus and minus components of the average parton momentum $\bar{k}=\left(k_{j}+k_{j}^{\prime}\right) / 2$ are given by

$$
k^{+}=\frac{1}{2 \sqrt{2}}[\sqrt{\hat{s}}+\sqrt{-\hat{u}}], \quad k^{-}=\frac{1}{2 \sqrt{2}}[\sqrt{\hat{s}}-\sqrt{-\hat{u}}] .
$$

Working out the matrix elements $\bar{u} Q_{i} u$ in the symmetric frame (results are listed in Table 1) and ignoring quark and meson masses, we can express the parton-level helicity amplitudes in terms of the invariant functions $C_{i}$ :

$$
\begin{aligned}
& \mathcal{H}_{0+,++}^{P(a b)}=\sqrt{-\hat{t} / 2} \hat{s}\left[C_{2}^{P(a b)}+C_{3}^{P(a b)}\right], \\
& \mathcal{H}_{0+,-+}^{P(a b)}=-\sqrt{-\hat{t} / 2} \hat{u}\left[C_{2}^{P(a b)}-C_{3}^{P(a b)}\right], \\
& \mathcal{H}_{0-,++}^{P(a b)}=-\sqrt{-\hat{u} \hat{s} / 2}\left[\hat{t} C_{1}^{P(a b)}-2 C_{4}^{P(a b)}\right], \\
& \mathcal{H}_{0-,-+}^{P(a b)}=\sqrt{-\hat{u} \hat{s} / 2} \hat{t} C_{1}^{P(a b)} .
\end{aligned}
$$

Other helicity amplitudes follow from Eq. (6). As inspection of (19) reveals, the invariant functions $C_{2}$ and $C_{3}$ contribute only to the quark helicity conserving amplitudes, while $C_{1}$ and $C_{4}$ generate quark helicity flips. As we discussed in Sect. 2, this feature entails different proton matrix elements, and hence different form factors, in the full process $\gamma B_{1} \rightarrow P B_{2}$.

\subsection{Subprocess observables}

As we will see in the next section, in the context of the handbag mechanism observables of the full process are often related to the corresponding ones for the subprocess in a simple fashion. It is therefore suitable to present some of the subprocess observables in terms of the invariant functions first.

An important observable for testing the handbag mechanism is the correlation between the helicities of the incoming photon and quark $[15,19]$. This observable is defined by

$$
\hat{A}_{L L}^{P(a b)}=\frac{\sum_{\lambda^{\prime}}\left\{\left|\mathcal{H}_{0 \lambda^{\prime},++}^{P(a b)}\right|^{2}-\left|\mathcal{H}_{0 \lambda^{\prime},+-}^{P(a b)}\right|^{2}\right\}}{\sum_{\lambda^{\prime}, \lambda}\left|\mathcal{H}_{0 \lambda^{\prime},+\lambda}^{P(a b)}\right|^{2}}
$$




\begin{tabular}{c||c|c} 
& $\lambda^{\prime}=\lambda$ & $\lambda^{\prime}=-\lambda$ \\
\hline \hline$Q_{1}$ & 0 & $-\sqrt{-\hat{u} \hat{s} / 2} \hat{t} \mu$ \\
$Q_{2}$ & $\frac{1}{2} \sqrt{-\hat{t} / 2}[(1+2 \lambda \mu) \hat{s}-(1-2 \lambda \mu) \hat{u}]$ & 0 \\
$Q_{3}$ & $\frac{1}{2} \sqrt{-\hat{t} / 2}[(1+2 \lambda \mu) \hat{s}+(1-2 \lambda \mu) \hat{u}]$ & 0 \\
$Q_{4}$ & 0 & $\sqrt{-\hat{u} \hat{s} / 2}(2 \lambda+\mu)$
\end{tabular}

Table 1: Matrix elements of the covariants $\bar{u}\left(k^{\prime}, \lambda^{\prime}\right) Q_{i} u(k, \lambda)$ defined in Eq. (16) evaluated in the symmetric frame specified by Eqs. (17) and (18).

and reads

$$
\begin{aligned}
\hat{A}_{L L}^{P(a b)}= & \left\{\left(\hat{s}^{2}-\hat{u}^{2}\right)\left[\left|C_{2}^{P(a b)}\right|^{2}+\left|C_{3}^{P(a b)}\right|^{2}\right]\right. \\
& +2\left(\hat{s}^{2}+\hat{u}^{2}\right) \operatorname{Re}\left(C_{2}^{P(a b)} C_{3}^{P(a b) *}\right) \\
& \left.+4 \frac{\hat{u} \hat{s}}{\hat{t}}\left|C_{4}^{P(a b)}\right|^{2}-4 \hat{u} \hat{s} \operatorname{Re}\left(C_{1}^{P(a b)} C_{4}^{P(a b) *}\right)\right\} / \hat{\mathcal{K}}^{P(a b)},
\end{aligned}
$$

in terms of the invariant functions. Here we have introduced the quantity

$$
\begin{aligned}
\hat{\mathcal{K}}^{P(a b)} & =\left(\hat{s}^{2}+\hat{u}^{2}\right)\left[\left|C_{2}^{P(a b)}\right|^{2}+\left|C_{3}^{P(a b)}\right|^{2}\right]+2\left(\hat{s}^{2}-\hat{u}^{2}\right) \operatorname{Re}\left(C_{2}^{P(a b)} C_{3}^{P(a b) *}\right) \\
& +2 \hat{t} \hat{u} \hat{s}\left|C_{1}^{P(a b)}\right|^{2}+4 \frac{\hat{u} \hat{s}}{\hat{t}}\left|C_{4}^{P(a b)}\right|^{2}-4 \hat{u} \hat{s} \operatorname{Re}\left(C_{1}^{P(a b)} C_{4}^{P(a b) *}\right),
\end{aligned}
$$

which, up to a factor, represents the sum over the squared absolute values of the subprocess helicity amplitudes.

One may also consider sideways polarisations of the quark where the $S$ direction is defined by $\mathbf{S}^{(\prime)}=\mathbf{N} \times \mathbf{L}^{(\prime)}$ for the incoming (outgoing) quark. $\mathbf{L}^{(\prime)}$ is the direction of the incoming (outgoing) quark's three-momentum and $\mathbf{N}=\mathbf{L} \times \mathbf{L}^{\prime}$ is the normal to the scattering plane. The correlation between the helicity of the incoming photon and the sideways polarisation of the incoming quark reads [15]

$$
\hat{A}_{L S}^{P(a b)}=2 \frac{\operatorname{Re}\left\{\mathcal{H}_{0+,++}^{P(a b)} \mathcal{H}_{0-,-+}^{P(a b) *}-\mathcal{H}_{0+,-+}^{P(a b)} \mathcal{H}_{0-,++}^{P(a b) *}\right\}}{\sum_{\lambda^{\prime}, \lambda}\left|\mathcal{H}_{0 \lambda^{\prime},+\lambda}^{P(a b)}\right|^{2}} .
$$

Expressing it in terms of the invariant functions, one finds

$$
\begin{aligned}
\hat{A}_{L S}^{P(a b)}= & 2 \sqrt{\frac{\hat{u} \hat{s}}{\hat{t}}} \operatorname{Re}\left\{\hat{t}(\hat{s}-\hat{u}) C_{2}^{P(a b)} C_{1}^{P(a b) *}-\hat{t}^{2} C_{3}^{P(a b)} C_{1}^{P(a b) *}\right. \\
& \left.+2 \hat{u}\left(C_{2}^{P(a b)}-C_{3}^{P(a b)}\right) C_{4}^{P(a b) *}\right\} / \hat{\mathcal{K}}^{P(a b)} .
\end{aligned}
$$

A non-zero $\hat{A}_{L S}^{P(a b)}$ requires both quark helicity flip (i.e., non-zero $C_{1}$ and/or $C_{4}$ ) and non-flip (i.e., non-zero $C_{2}$ and/or $C_{3}$ ) amplitudes. 
As we see, the observables in general depend on the invariant functions in a complicated way. If one of the invariant functions dominates the others, the expressions $(21,22,24)$ become simple and clear and exhibit differences characteristic of the dominating invariant function. If, for instance, either $C_{2}$ or $C_{3}$ dominates (or if their phases differ by $90^{\circ}$ and $C_{1}$, $C_{4}$ are negligible), $\hat{A}_{L L}^{P(a b)}$ reduces to

$$
\hat{A}_{L L}^{P(a b)} \simeq \frac{\hat{s}^{2}-\hat{u}^{2}}{\hat{s}^{2}+\hat{u}^{2}}
$$

The result (25) coincides with the helicity correlation in $\gamma q \rightarrow \gamma q$, the subprocess occurring in Compton scattering [15]. In other cases, the helicity correlation differs from (25) drastically. Thus, for instance, if $C_{1}$ dominates, $\hat{A}_{L L}^{P(a b)}$ is zero, while a dominant $C_{4}$ leads to $\hat{A}_{L L}^{P(a b)}=$ 1. How these subprocess results affect the full process, namely meson photoproduction off baryons, remains to be discussed.

\subsection{Contributions from twist-2 and twist-3 meson generation}

As an example of a dynamical scenario, let us discuss the one-gluon exchange mechanism. The expressions obtained from the Feynman graphs shown in Fig. 2 are to be convoluted with the meson distribution amplitude. According to Beneke and Feldmann [20], the light-cone projection operator of an outgoing pseudoscalar meson in momentum space, including twist-3 two-particle contributions, reads (conveniently rewritten in the notation of [21])

$$
\begin{aligned}
\mathcal{P}_{\alpha \beta, k l}^{P(a b)=} & \frac{f_{P}}{2 \sqrt{2 N_{C}}} \mathcal{C}_{P}^{a b} \frac{\delta_{k l}}{\sqrt{N_{C}}}\left\{\frac{\gamma_{5}}{\sqrt{2}} \not q^{\prime} \phi_{P}(\tau)+\mu_{P} \frac{\gamma_{5}}{\sqrt{2}}\left[\phi_{P p}(\tau)\right.\right. \\
& \left.\left.-i \sigma_{\mu \nu} \frac{q^{\prime \mu} k^{\prime \nu}}{q^{\prime} \cdot k^{\prime}} \frac{\phi_{P \sigma}^{\prime}(\tau)}{6}+i \sigma_{\mu \nu} q^{\prime \mu} \frac{\phi_{P \sigma}(\tau)}{6} \frac{\partial}{\partial \ell_{\perp \nu}}\right]\right\}_{\alpha \beta},
\end{aligned}
$$

where $f_{P}$ is the meson's decay constant; for instance, $f_{\pi}=132 \mathrm{MeV} ; \alpha(a, k)$ and $\beta(b$, $l$ ) represent Dirac (flavour, colour) labels of the quark and antiquark, respectively. The parameter $\mu_{P}$ in (26) is proportional to the chiral condensate. For the pion, the familiar result is $\mu_{\pi}=m_{\pi}^{2} /\left(m_{u}+m_{d}\right) \simeq 2 \mathrm{GeV}$ at a scale of $2 \mathrm{GeV}$. In (26), $\ell_{\perp}$ denotes the transverse momentum of the quark entering the meson, defined with respect to the meson's momentum, $q^{\prime}$. After performing the derivative the collinear limit, $\ell_{\perp}=0$, is taken. Note that in the massless limit we are working in, the two vectors $q^{\prime}$ and $k^{\prime} /\left(k^{\prime} \cdot q^{\prime}\right)$ are light-like and their space components have opposite sign ${ }^{1}$. The projector takes into account the familiar twist-2 distribution amplitude $\phi_{P}(\tau)$ and the two-particle twist-3 ones $\phi_{P p}(\tau)$ and $\phi_{P \sigma}(\tau)$, where $\tau$ is the momentum fraction carried by the quark inside the meson. In $(26), \phi_{P \sigma}^{\prime}$ denotes the derivative of $\phi_{P \sigma}$ with respect to $\tau$. A complete projector to twist-3 accuracy would also include three-particle contributions [22]. Assuming the three-particle distributions to be strictly zero, the equations of motion fix the twist-3 distribution amplitudes $[20,22]$ to:

$$
\phi_{P p}(\tau)=1, \quad \phi_{P \sigma}(\tau)=6 \tau(1-\tau) .
$$

Although strictly vanishing three-particle distributions cannot be justified [20], the form (27) gives a hint at the magnitude of the twist-3 two-particle contribution. In $[22,23]$ the next

\footnotetext{
${ }^{1}$ In the frame where $q^{\prime}=\left(q^{\prime+}, 0, \mathbf{0}_{\perp}\right), q^{\prime \mu} k^{\prime \nu} /\left(k^{\prime} \cdot q^{\prime}\right)=q^{\prime \mu} n^{\nu} /\left(n \cdot q^{\prime}\right)$ where $n=\left(0,1, \mathbf{0}_{\perp}\right)$ and $q^{\prime} \cdot n=q^{\prime+}$.
} 
Gegenbauer coefficients for $\phi_{P p}$ and $\phi_{P \sigma}$ have been calculated exploiting results for the threeparticle twist-3 distribution amplitudes and equations of motion.

The explicit calculation of the leading-order twist-2 contribution to the process $\gamma q_{a} \rightarrow P q_{b}$ provides the following results for the invariant functions:

$$
\begin{aligned}
\left.C_{2}^{P(a b)}\right|_{t w i s t-2} & =-2 \pi \alpha_{s}\left(\mu_{R}^{2}\right) f_{P} \mathcal{C}_{P}^{a b} \frac{C_{F}}{N_{c}} \frac{e_{a} \hat{u}+e_{b} \hat{s}}{\hat{t}} \frac{\langle 1 /(1-\tau)\rangle_{P}+\langle 1 / \tau\rangle_{P}}{\hat{u} \hat{s}}, \\
\left.C_{3}^{P(a b)}\right|_{t w i s t-2} & =-2 \pi \alpha_{s}\left(\mu_{R}^{2}\right) f_{P} \mathcal{C}_{P}^{a b} \frac{C_{F}}{N_{c}} \frac{e_{a} \hat{u}+e_{b} \hat{s}}{\hat{t}} \frac{\langle 1 /(1-\tau)\rangle_{P}-\langle 1 / \tau\rangle_{P}}{\hat{u} \hat{s}}, \\
\left.C_{1}^{P(a b)}\right|_{\text {twist-2 }} & =\left.C_{4}^{P(a b)}\right|_{\text {twist-2 }}=0 .
\end{aligned}
$$

Here, $\mu_{R}$ is an appropriate renormalisation scale and $C_{F}=\left(N_{c}^{2}-1\right) /\left(2 N_{c}\right)$ is the usual $\mathrm{SU}(3)$ colour factor. Note that the positron charge $e$ has been pulled out of the subprocess amplitudes and is written explicitly in (4) and (11). The flavour weight factors $\mathcal{C}_{P}^{a b}$ comprise the flavour structure of the meson. For pions they read

$$
\mathcal{C}_{\pi^{0}}^{u u}=-\mathcal{C}_{\pi^{0}}^{d d}=1 / \sqrt{2}, \quad \mathcal{C}_{\pi^{+}}^{u d}=\mathcal{C}_{\pi^{-}}^{d u}=1,
$$

all other factors are zero, (e.g., $\mathcal{C}_{\pi^{0}}^{s s}=0$ ), since the projection operator (26) implies the valence quark approximation for the meson. $\langle 1 / \tau\rangle_{P}$ and $\langle 1 /(1-\tau)\rangle_{P}$ are moments of the meson's twist-2 distribution amplitude

$$
\langle 1 / \tau\rangle_{P}=\int_{0}^{1} d \tau \frac{\phi_{P}(\tau)}{\tau} .
$$

Owing to the evolution of the distribution amplitudes the moments depend on the factorisation scale $\mu_{F}$. The pion distribution amplitude is symmetric under the replacement $\tau \leftrightarrow 1-\tau$; hence $C_{3}^{\pi}$ is zero as well. For kaons, for instance, this symmetry does not hold.

To leading-order in $\alpha_{s}$ the twist-3 content of the projector (26) leads to the invariant functions

$$
\begin{aligned}
\left.C_{1}^{P(a b)}\right|_{t w i s t-3} & =-4 \pi \alpha_{s}\left(\mu_{R}^{2}\right) \frac{C_{F}}{N_{C}} \frac{f_{P} \mu_{P}}{\hat{t}} \mathcal{C}_{P}^{a b} \int_{0}^{1} d \tau \\
& \times\left\{\frac{e_{a}}{\hat{s}^{2}}\left[-\frac{\phi_{P p}}{1-\tau}-\left(\frac{2}{\tau}+\frac{1}{1-\tau}\right) \frac{\phi_{P \sigma}^{\prime}}{6}+\frac{\phi_{P \sigma}}{3 \tau^{2}}\right]\right. \\
& +\frac{e_{b}}{\hat{u}^{2}}\left[-\frac{\phi_{P p}}{\tau}+\left(\frac{2}{1-\tau}+\frac{1}{\tau}\right) \frac{\phi_{P \sigma}^{\prime}}{6}+\frac{\phi_{P \sigma}}{3(1-\tau)^{2}}\right] \\
& \left.+\frac{e_{b} \hat{t}}{\hat{s} \hat{u}^{2}}\left[\frac{\phi_{P p}}{\tau}+\left(\frac{2}{1-\tau}+\frac{1}{\tau}\right) \frac{\phi_{P \sigma}^{\prime}}{6}-\frac{\left(1-\tau-\tau^{2}\right)}{\tau^{2}(1-\tau)^{2}} \frac{\phi_{P \sigma}}{3}\right]\right\},
\end{aligned}
$$

and

$$
\begin{aligned}
\left.C_{4}^{P(a b)}\right|_{t w i s t-3}= & -4 \pi \alpha_{s}\left(\mu_{R}^{2}\right) \frac{C_{F}}{N_{C}} f_{P} \mu_{P} \mathcal{C}_{P}^{a b} \int_{0}^{1} d \tau \\
\times & \left\{\frac{e_{a}}{\hat{s}^{2}}\left[-\frac{\phi_{P p}}{1-\tau}-\frac{\phi_{P \sigma}^{\prime}}{6 \tau(1-\tau)}+\frac{\phi_{P \sigma}}{6 \tau^{2}(1-\tau)}\right]\right. \\
& +\frac{e_{b}}{\hat{u}^{2}}\left[-\frac{\phi_{P p}}{\tau}+\frac{\phi_{P \sigma}^{\prime}}{6 \tau(1-\tau)}+\frac{\phi_{P \sigma}}{6 \tau(1-\tau)^{2}}\right] \\
& \left.+\frac{e_{b} \hat{t}}{\hat{s} \hat{u}^{2}}\left[\frac{\phi_{P \sigma}^{\prime}}{6 \tau(1-\tau)}-\frac{1-2 \tau}{\tau^{2}(1-\tau)^{2}} \frac{\phi_{P \sigma}}{6}\right]\right\} .
\end{aligned}
$$


Furthermore,

$$
\left.C_{2}^{P(a b)}\right|_{\text {twist-3 }}=\left.C_{3}^{P(a b)}\right|_{\text {twist }-3}=0 .
$$

Inserting the distribution amplitudes (27) into Eqs. (31) and (32), we obtain

$$
\left.C_{1}^{P(a b)}\right|_{\text {twist-3 }}=\left.C_{4}^{P(a b)}\right|_{\text {twist-3 }}=0 .
$$

Although (34) is a consequence of the special distribution amplitudes (27) we take it as a hint at the smallness of the twist-3 contribution. The generalisation to more general distribution amplitudes than (27) is beyond the scope of the present paper and is left to a forthcoming publication. Such a calculation requires the inclusion of three-particle twist-3 contributions for consistency. Here we only remark that the distribution amplitudes proposed in [22, 23] lead to finite results for $C_{1}^{P(a b)}$ and $C_{4}^{P(a b)}$. In particular, as can easily be seen, factorisation holds for them ${ }^{2}$.

Perturbative corrections to the twist- 2 and twist-3 contributions will not change the separation of quark helicity flip and non-flip. Each additional gluon goes along with an even number of $\gamma$ matrices. Therefore, there is no mixing between $C_{2}$ and $C_{3}$, on the one side, and $C_{1}$ and $C_{4}$ on the other side.

\section{Predictions from the handbag mechanism for meson photoproduction}

\subsection{General remarks}

The twist-2 contribution (28) from the handbag mechanism to pion photoproduction has been computed in [4]. Using the asymptotic distribution amplitude $\phi_{\pi}(\tau)=6 \tau(1-\tau)$, leading to a value of 3 for the $1 / \tau$ moment, a cross section has been obtained far below experiment. We have also seen that the contribution with the twist-3 meson distribution amplitude does not solve this problem. It is suggestive to assume that a more general mechanism, unknown at present, is at work for the generation of the meson. It may consist of a resummation of perturbative corrections and/or a sum over higher twists or power corrections. There is, of course, also the possibility that the handbag contribution does not dominate meson photoproduction at momentum transfer of the order of $10 \mathrm{GeV}^{2}$.

In this section we discuss predictions for meson photoproduction from the handbag approach without assuming a specific mechanism for the generation of the meson. Supported by our estimates of the twist-3 contributions, we conjecture the suppression of quark helicity flip. In the handbag mechanism the baryons emit and reabsorb partons which carry momentum fractions close to unity (see Sect. 2). From phenomenological and theoretical considerations we expect these partons to be most likely valence quarks of the baryons; sea quarks are disfavoured. Hence, in order to simplify our analysis further, we assume the active partons to be valence quarks of the involved baryons.

Experimental verification of various predictions we present below, would be a clear hint at the dominance of the handbag mechanism and will shed light on the dynamics of the meson generation. Provided the form factors $R_{i}^{P}$ are sufficiently well-known which will likely be the case soon owing to the running JLab measurements on Compton scattering and the

\footnotetext{
${ }^{2}$ This statement is only correct if the chiral corrections advocated for in [23], are neglected. These terms break factorisation.
} 
universality property of the GPDs, it might even be possible to determine the invariant functions $C_{2}^{P}$ and $C_{3}^{P}$ directly from experiment. Despite the still not understood normalisation of the cross section, we believe that the experimental examination of the handbag predictions is a rewarding goal.

To start with, we consider the flavour dependence of the invariant functions $C_{i}^{P(a b)}$ for the production of charged pions. In order to understand this dependence, it is instructive to make the isovector $C_{i}^{(-)}$and isoscalar $C_{i}^{(0)}$ content of the invariant functions explicit by writing [18]

$$
\begin{aligned}
C_{i}^{(-)} & =\frac{1}{2 \sqrt{2}}\left[C_{i}^{\pi^{+}}-C_{i}^{\pi^{-}}\right] \\
C_{i}^{(0)} & =\frac{1}{2 \sqrt{2}}\left[C_{i}^{\pi^{+}}+C_{i}^{\pi^{-}}\right],
\end{aligned}
$$

where the flavour superscripts $u d, d u$ are omitted since there is only one subprocess. $C_{2}^{(-)}$is antisymmetric under the $\hat{s} \leftrightarrow \hat{u}$ crossing, while $C_{2}^{(0)}$ is symmetric [18]. Using these crossing properties and charge factors corresponding to isovector and isoscalar combinations of $u$ and $d$ quarks, we write ${ }^{3}$

$$
\begin{aligned}
C_{2}^{(-)} & =\left(e_{u}-e_{d}\right) \frac{\hat{s}-\hat{u}}{\hat{t}} \frac{c_{2}(\hat{s}, \hat{t})}{2 \sqrt{2}}, \\
C_{2}^{(0)} & =\left(e_{u}+e_{d}\right) \frac{c_{2}(\hat{s}, \hat{t})}{2 \sqrt{2}},
\end{aligned}
$$

where we assume isospin independence of the reduced invariant function $c_{2}$. It is symmetric under the $\hat{s} \leftrightarrow \hat{u}$ crossing. Hence,

$$
\begin{aligned}
& C_{2}^{\pi^{+}}(\hat{s}, \hat{t})=\frac{e_{u} \hat{u}+e_{d} \hat{s}}{\hat{s}+\hat{u}} c_{2}(\hat{s}, \hat{t}), \\
& C_{2}^{\pi^{-}}(\hat{s}, \hat{t})=\frac{e_{d} \hat{u}+e_{u} \hat{s}}{\hat{s}+\hat{u}} c_{2}(\hat{s}, \hat{t}),
\end{aligned}
$$

in conformity with the twist-2 contribution (28). The associated form factors $R_{i, p(n) \rightarrow n(p)}^{u d(d u)}=$ $R^{\pi^{ \pm}}$are given in (10).

The invariant function $C_{3}^{\pi}$ exhibits a crossing behaviour opposite to that of $C_{2}^{\pi}$ [18]. Therefore, we assume in analogy to (36)

$$
\begin{aligned}
C_{3}^{(-)} & =\left(e_{u}-e_{d}\right) \frac{c_{3}(\hat{s}, \hat{t})}{2 \sqrt{2}}, \\
C_{3}^{(0)} & =\left(e_{u}+e_{d}\right) \frac{\hat{s}-\hat{u}}{\hat{t}} \frac{c_{3}(\hat{s}, \hat{t})}{2 \sqrt{2}},
\end{aligned}
$$

which leads to

$$
\begin{aligned}
C_{3}^{\pi^{+}}(\hat{s}, \hat{t}) & =\frac{e_{u} \hat{u}-e_{d} \hat{s}}{\hat{s}+\hat{u}} c_{3}(\hat{s}, \hat{t}), \\
C_{3}^{\pi^{-}}(\hat{s}, \hat{t}) & =\frac{e_{d} \hat{u}-e_{u} \hat{s}}{\hat{s}+\hat{u}} c_{3}(\hat{s}, \hat{t}) .
\end{aligned}
$$

\footnotetext{
${ }^{3}$ Since we are working in the massless limit, $(\hat{s}-\hat{u}) / \hat{t}$ is, up to $\hat{s} \leftrightarrow \hat{u}$ symmetric factors, the only possibility to construct a dimensionless crossing-odd term.
} 
The reduced invariant function $c_{3}$ is crossing symmetric as $c_{2}$.

For $\pi^{0}$ photoproduction we have to consider two subprocesses $\gamma u \rightarrow \pi^{0} u$ and $\gamma d \rightarrow \pi^{0} d$. According to [18] their isospin decomposition involves the isoscalar part $C_{i}^{(0)}$, which also appears in the charged pion case, and a new isovector component $C_{i}^{(+)}$which has the same crossing properties as $C_{i}^{(0)}$. In analogy to (36), (38) and assuming again isospin independence of the reduced invariant functions we write

$$
\begin{aligned}
C_{2}^{(+)} & =\left(e_{u}-e_{d}\right) \frac{c_{2}(\hat{s}, \hat{t})}{2 \sqrt{2}}, \\
C_{3}^{(+)} & =\left(e_{u}-e_{d}\right) \frac{\hat{s}-\hat{u}}{\hat{t}} \frac{c_{3}(\hat{s}, \hat{t})}{2 \sqrt{2}} .
\end{aligned}
$$

Sum and difference of $C_{i}^{(0)}$ and $C_{i}^{(+)}$provide the subprocess amplitudes for the $\pi^{0}$ photoproduction $(a=u, d)$

$$
\begin{aligned}
C_{2}^{\pi^{0}(a a)}(\hat{s}, \hat{t}) & =e_{a} \mathcal{C}_{\pi^{0}}^{a a} c_{2}(\hat{s}, \hat{t}) \\
C_{3}^{\pi^{0}(a a)}(\hat{s}, \hat{t}) & =e_{a} \mathcal{C}_{\pi^{0}}^{a a} \frac{\hat{s}-\hat{u}}{\hat{t}} c_{3}(\hat{s}, \hat{t})
\end{aligned}
$$

where the $\mathcal{C}_{\pi^{0}}^{a a}$ are the flavour weight factors defined in (29). The leading-twist result (28) is again in agreement with these isospin and crossing considerations. Note that in contrast to the case of charged pions, the flavour dependence here appears as a constant factor. It is therefore convenient, following previous work $[4,5,13]$, to pull out the flavour dependence $e_{a} \mathcal{C}_{\pi^{0}}^{a a}$ from the subprocess amplitudes and to absorb them into the form factors which leads to

$$
R_{i}^{\pi^{0}}=e_{u} \mathcal{C}_{\pi^{0}}^{u u} R_{i}^{u}+e_{d} \mathcal{C}_{\pi^{0}}^{d d} R_{i}^{d}=\frac{1}{\sqrt{2}}\left(e_{u} R_{i}^{u}-e_{d} R_{i}^{d}\right)
$$

The remaining subprocess amplitudes for which the flavour labels are to be dropped now, read

$$
C_{2}^{\pi^{0}}=c_{2}(\hat{s}, \hat{t}), \quad C_{3}^{\pi^{0}}=\frac{\hat{u}-\hat{s}}{\hat{s}+\hat{u}} c_{3}(\hat{s}, \hat{t}) .
$$

For subsequent numerical studies, a model for the form factors or for the underlying GPDs is required. Following $[4,13,15]$, we use a model which is motivated by overlaps of light-cone wave functions $[12,13,24]$ and is designed for large $-t$ and zero skewness:

$$
\begin{aligned}
H^{a}(x, 0 ; t) & =\exp \left[a_{N}^{2} t \frac{1-x}{2 x}\right]\left[q_{a}(x)-\bar{q}_{a}(x)\right], \\
\widetilde{H}^{a}(x, 0 ; t) & =\exp \left[a_{N}^{2} t \frac{1-x}{2 x}\right]\left[\Delta q_{a}(x)-\Delta \bar{q}_{a}(x)\right],
\end{aligned}
$$

where $q(x)$ and $\Delta q(x)$ are the usual unpolarised and polarised parton distributions in the proton ${ }^{4,5}$. Note that here we display quarks and antiquarks explicitly; therefore, the moments of the GPDs are obtained from them by integrating only from $x=0$ to 1 . Forced by the Gaussian in (44), only large $x$ contribute for large $-t$. For the transverse size parameter of the proton $a_{N}$, we take a value of $0.8 \mathrm{GeV}$ [13]. Since the phenomenological parton distributions

\footnotetext{
${ }^{4}$ In the full process for which the GPDs are needed, $t$ is identified with $\hat{t}$.

${ }^{5}$ In principle, the helicity-flip GPD $H_{T}^{a}$ can be modelled analogously to (44). In this case, the corresponding PDFs are the transversity distributions $\delta^{a}(x)$.
} 
[25] suffer from large uncertainties at large $x$, which is an important region for hard wide-angle scattering, the model is to be improved at large $x$, see $[13,26]$.

The form factor $R_{T}$ is not modelled explicitly but rather fixed relative to $R_{V}$. According to a recent measurement performed at JLab [27], its electromagnetic analogue, the Pauli form factor of the proton $F_{2}$, seems to be suppressed by $\Lambda / \sqrt{-t}$ as compared to the Dirac form factor $F_{1}$. Since, on the one hand, $F_{1}$ and $R_{V}$ are related to the GPD $H$, while, on the other hand, $F_{2}$ and $R_{T}$ are associated with $E$, it is plausible to expect a similar suppression of $R_{T}$, as seems to occur for $F_{2}$. Hence, as has been discussed in [15], the ratio

$$
\kappa_{T}^{P}=\frac{\sqrt{-t}}{2 m} \frac{R_{T}^{P}}{R_{V}^{P}}
$$

may be expected to be a constant at large $-t$ and $R_{T}$ and, hence, the proton helicity flip amplitude (5), has therefore to be taken into account. However, a suppression of $R_{T}$ like $\Lambda^{2} / t$ cannot be excluded as yet [28], although recent estimates [29] of two-photon exchange contributions seem to favour the JLab results [27]. In the former case, one may ignore $R_{T}$ and proton helicity flip for not too small values of $-t$.

\subsection{Ratio of $\pi^{+}$and $\pi^{-}$photoproduction cross sections}

The derivation of the photoproduction amplitudes within the handbag approach naturally requires the use of the light-cone helicity basis. However, for comparison with experimental and other theoretical results, the use of the ordinary photon-nucleon c.m.s. helicity basis is more convenient. The standard helicity amplitudes $\Phi_{0 \nu^{\prime}, \mu \nu}$, defined in a c.m.s. in which the photon and the incoming proton move along the 3-direction, are obtained from the light-cone helicity amplitudes (4), defined in the symmetric frame, by the following transform [15, 14]:

$$
\Phi_{0 \nu^{\prime}, \mu \nu}^{P}=\mathcal{M}_{0 \nu^{\prime}, \mu \nu}^{P}+\beta / 2\left[(-1)^{1 / 2-\nu^{\prime}} \mathcal{M}_{0-\nu^{\prime}, \mu \nu}^{P}+(-1)^{1 / 2+\nu} \mathcal{M}_{0 \nu^{\prime}, \mu-\nu}^{P}\right],
$$

where

$$
\beta=\frac{2 m}{\sqrt{s}} \frac{\sqrt{-t}}{\sqrt{s}+\sqrt{-u}} .
$$

Since we are now discussing the full process within the handbag approach, we can make use of Eq. (3). As pointed out in Ref. [30], besides this there are ambiguities in relating the massless kinematics used in the handbag approach with the experimental one for which, in particular at energies available at JLab, the proton mass cannot be ignored. For the numerical results presented here we use the identification of our Mandelstam variables with the experimental ones $\left(s_{\exp }, u_{\exp }, t_{\exp }\right)$ in which the nucleon mass is taken into account properly (scenario 2 in the terminology of [30])

$$
s=s_{\text {exp }}-m^{2}, \quad t=t_{\exp }, \quad u=u_{\exp }-m^{2} .
$$

The unpolarised differential cross section for the production of a pseudoscalar meson is given by

$$
\frac{d \sigma^{P}}{d t}=\frac{1}{32 \pi s^{2}} \sum_{\nu^{\prime}, \mu}\left|\Phi_{0 \nu^{\prime}, \mu+}^{P}\right|^{2},
$$

where the sum over the squared helicity amplitudes can be expressed as

$$
\sum_{\nu^{\prime}, \mu}\left|\Phi_{0 \nu^{\prime}, \mu+}^{P}\right|^{2}=-\left(\frac{e}{2}\right)^{2} t(s-u)^{2} \mathcal{K}^{P} .
$$




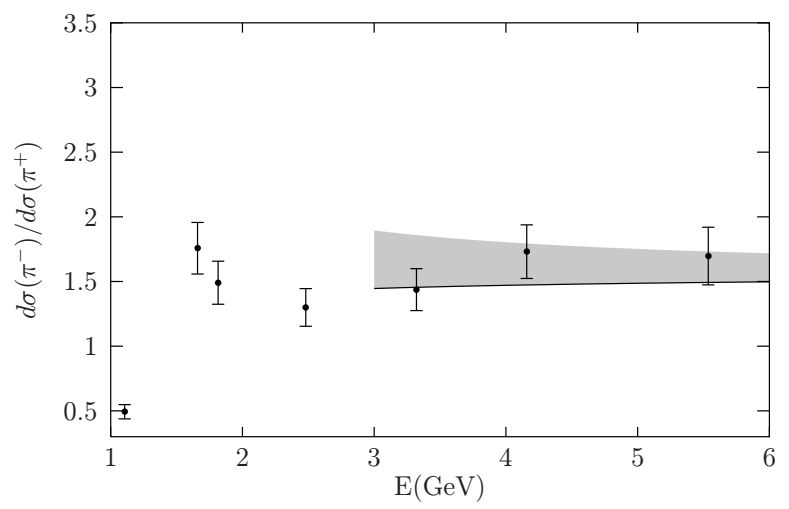

Figure 4: The ratio of the $\gamma n \rightarrow \pi^{-} p$ and $\gamma p \rightarrow \pi^{+} n$ cross sections versus photon beam energy $E$, at a c.m.s. scattering angle of $90^{\circ}$. Data are taken from [31]. The solid line is the handbag prediction with the identification (48). The uncertainties due to target mass corrections [30] are indicated by the shaded band.

For photoproduction of $\pi$ mesons, the function $\mathcal{K}^{P}$ is defined by

$$
\begin{aligned}
\mathcal{K}^{P}= & {\left[\left(R_{V}^{P}\right)^{2}\left[1+\left(\kappa_{T}^{P}\right)^{2}\right]+\frac{t^{2}}{(s-u)^{2}}\left(R_{A}^{P}\right)^{2}\right]\left|C_{2}^{P}\right|^{2} } \\
& +\left[\frac{t^{2}}{(s-u)^{2}}\left(R_{V}^{P}\right)^{2}\left[1+\left(\kappa_{T}^{P}\right)^{2}\right]+\left(R_{A}^{P}\right)^{2}\right]\left|C_{3}^{P}\right|^{2} \\
& -\frac{t}{s-u}\left[\left(R_{V}^{P}\right)^{2}\left[1+\left(\kappa_{T}^{P}\right)^{2}\right]+\left(R_{A}^{P}\right)^{2}\right] 2 \operatorname{Re}\left(C_{2}^{P *} C_{3}^{P}\right),
\end{aligned}
$$

if $C_{1}^{P}$ and $C_{4}^{P}$ are neglected.

If $C_{2}^{P}$ dominates, we obtain from (37) and (51) for the ratio of $\pi^{ \pm}$cross sections ${ }^{6}$

$$
\frac{d \sigma\left(\gamma n \rightarrow \pi^{-} p\right)}{d \sigma\left(\gamma p \rightarrow \pi^{+} n\right)}=\left(\frac{e_{u} s+e_{d} u}{e_{u} u+e_{d} s}\right)^{2}
$$

As Fig. 4 reveals, the prediction (52) is in surprisingly good agreement with recent experimental results from JLab [31] given the small photon beam energies $\left(s_{\text {exp }}=2 m E+m^{2}\right)$. For comparison, we also quote the result for the ratio of cross section which follows from the dominance of $C_{3}^{P}$. In this case, we find from (39) and (51)

$$
\frac{d \sigma\left(\gamma n \rightarrow \pi^{-} p\right)}{d \sigma\left(\gamma p \rightarrow \pi^{+} n\right)}=\left(\frac{e_{u} s-e_{d} u}{e_{u} u-e_{d} s}\right)^{2}
$$

which tends to infinity at large $s$ and a scattering angle of $90^{\circ}$ and is clearly at variance with experiment [31]. For the special case of equal reduced invariant functions, $c_{2}=c_{3}$, the ratio of cross sections is unity. Thus, there is a strong indication from experiment that the handbag mechanism is at work in these processes with $\left|C_{2}^{P}\right| \gg\left|C_{3}^{P}\right|$ provided our assumption of negligible quark helicity flip contributions holds. Structurally, the result (52) coincides with the leading-twist prediction with, however, a more general function $C_{2}^{P}$.

\footnotetext{
${ }^{6}$ The invariant functions $C_{1}^{(0,-)}$ and $C_{4}^{(0,-)}$ have the same crossing properties as $C_{2}^{(0,-)}$ and would therefore lead to the same ratio of $\pi^{-}$and $\pi^{+}$cross sections.
} 


\subsection{Spin correlations}

Spin correlations provide further severe tests of the handbag mechanism. They are given by ratios of partial cross sections within one and the same process and are, therefore, independent of flavour symmetry breaking effects. If one of the invariant functions dominates, for which the ratio of $\pi^{ \pm}$cross sections provides some evidence, it cancels and an absolute prediction for spin correlations is obtained.

The correlation of the incoming photon and nucleon helicities, $A_{L L}$, or the helicity transfer from the incoming photon to the outgoing nucleon $K_{L L}$, is defined as in (20) but with the subprocess amplitudes, $\mathcal{H}$, replaced by the corresponding amplitudes, $\Phi$, of the full process. In terms of form factors and invariant functions, the helicity correlation for pion photoproduction reads

$$
\begin{aligned}
A_{L L}^{P} & =K_{L L}^{P}=\frac{-2 t}{s-u} \frac{R_{A}^{P} R_{V}^{P}}{\mathcal{K}^{P}}\left(1+\beta \kappa_{T}^{P}\right) \\
& \times\left[\left|C_{2}^{P}\right|^{2}+\left|C_{3}^{P}\right|^{2}+2 \frac{s^{2}+u^{2}}{s^{2}-u^{2}} \operatorname{Re}\left(C_{2}^{P *} C_{3}^{P}\right)\right],
\end{aligned}
$$

if $C_{1}^{P}$ and $C_{4}^{P}$ are neglected. The expression strongly simplifies if one of the invariant functions dominates and if some reasonable kinematical approximations as well as the fact that $R_{A}^{2} \leq R_{V}^{2}$, are used. The latter property follows from (44) directly. Thus, if $C_{2}^{P} \gg C_{3}^{P}$, one finds

$$
A_{L L}^{P} \simeq \frac{s^{2}-u^{2}}{s^{2}+u^{2}} \frac{R_{A}^{P}}{R_{V}^{P}}\left(1+\beta \kappa_{T}^{P}\right),
$$

while for $C_{3}^{P} \gg C_{2}^{P}$, the roles of $R_{V}^{P}$ and $R_{A}^{P}$ are roughly interchanged. Equation (55) is the same expression as one finds in wide-angle Compton scattering [15]. Provided one of the invariant functions dominates, the helicity correlation should exhibit a scattering angle dependence as that of the corresponding observable for the partonic subprocess (25); only its magnitude is diluted by a ratio of slightly process-dependent form factors, see Fig. 5. If, on the other hand, the reduced invariant functions (43) have roughly the same value, $c_{2} \simeq c_{3}$, one obtains $A_{L L}^{\pi^{0}} \simeq 0$, while the helicity correlations for charged pions are non-zero but small in magnitude. In Fig. 6 we display results for the helicity correlation in $\pi^{0}$ photoproduction obtained for three different assumptions on the reduced invariant functions $c_{2}$ and $c_{3}$ in order to demonstrate the sensitivity of this observable to the underlying meson generation mechanism.

The correlation between the helicity of the incoming photon and the sideways polarisation of either the incoming $\left(A_{L S}\right)$ or outgoing $\left(K_{L S}\right)$ nucleon is defined as in (23) but, as for $A_{L L}$, with the $\mathcal{H}$ being replaced by the amplitudes $\Phi$. This definition leads to $\left(C_{1}^{P}, C_{4}^{P}\right.$ neglected)

$$
\begin{aligned}
A_{L S}^{P}= & -K_{L S}^{P}=\frac{2 t}{s-u} \frac{R_{V}^{P} R_{A}^{P}}{\mathcal{K}^{P}}\left(\beta-\kappa_{T}^{P}\right) \\
& {\left[\left|C_{2}^{P}\right|^{2}+\left|C_{3}^{P}\right|^{2}+2 \frac{s^{2}+u^{2}}{s^{2}-u^{2}} \operatorname{Re}\left(C_{2}^{P} C_{3}^{P *}\right)\right] . }
\end{aligned}
$$

Predictions for $A_{L S}$ are shown in Fig. 7. If $C_{2}^{P} \gg C_{3}^{P}$, for instance, (56) simplifies to

$$
A_{L S}^{P} \simeq \frac{s^{2}-u^{2}}{s^{2}+u^{2}} \frac{R_{A}^{P}}{R_{V}^{P}}\left(\kappa_{T}^{P}-\beta\right),
$$




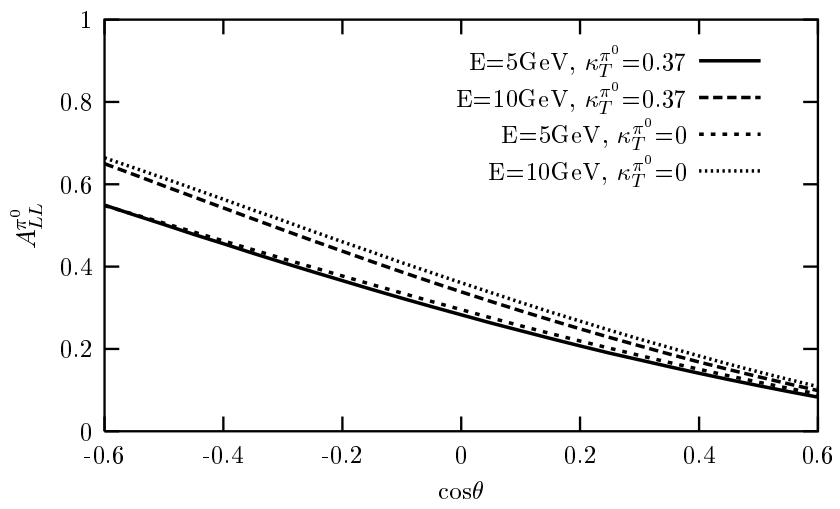

Figure 5: Handbag predictions for the helicity correlation (54) in $\pi^{0}$ photoproduction vs. $\cos \theta$ at two different beam energies and two values of the ratio $\kappa_{T}^{\pi^{0}}$ assuming the dominance of the invariant function $C_{2}^{\pi^{0}}$. Target mass corrections are not shown.

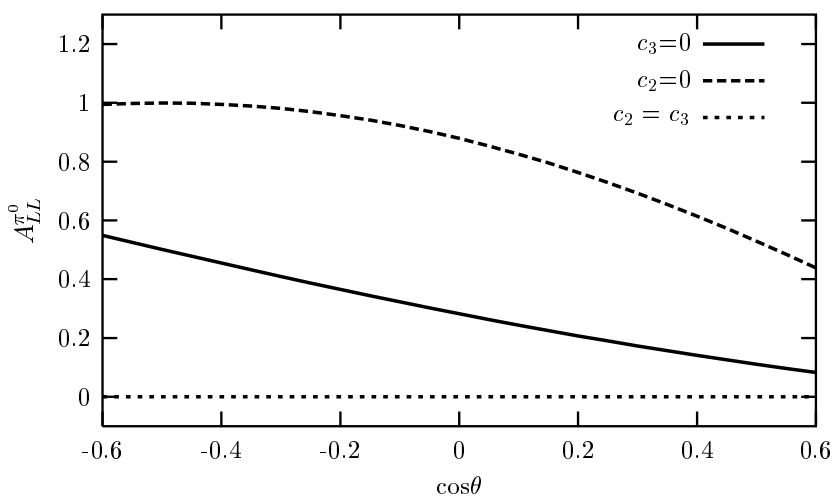

Figure 6: Handbag predictions for the helicity correlation $A_{L L}$ (54) in $\pi^{0}$ photoproduction vs. $\cos \theta$ at a beam energy of $5 \mathrm{GeV}$ and with $\kappa_{T}^{\pi^{0}}=0.37$. For the invariant functions (43), the three cases $c_{3}=0, c_{2}=0$, and $c_{3}=c_{2}$ are investigated. 


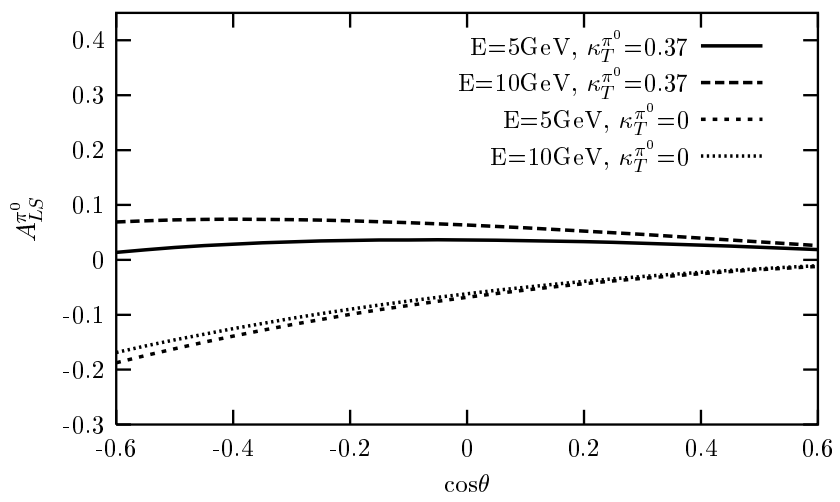

Figure 7: The helicity correlation $A_{L S}(56)$ for $\pi^{0}$ photoproduction. For the legend, see Fig. 5.

and a similar expression in the case of a dominant $C_{3}^{P}$. In contrast to $A_{L L}^{P}$, which is a rather robust prediction of the handbag approach, $A_{L S}^{P}$ depends on the difference of two correction terms: the kinematical factor $\beta$ which controls the transform of the light-cone helicity amplitudes to the standard c.m.s. ones and the badly known tensor form factor. Hence, one has to be aware of possible large corrections to $A_{L S}^{P}$. We also repeat that the subprocess $\hat{A}_{L S}^{P(a b)}$ is zero if quark helicity flip is neglected, see (24), which again signals that $A_{L S}^{P}$ is dominated by soft physics.

A particular noteworthy result is the ratio of the two correlation parameters

$$
\frac{A_{L S}^{P}}{A_{L L}^{P}}=-\frac{K_{L S}^{P}}{K_{L L}^{P}}=\frac{\kappa_{T}^{P}-\beta}{1+\beta \kappa_{T}^{P}}
$$

which holds for any values of $C_{2}^{P}$ and $C_{3}^{P}$ as can easily be seen from (54) and (56). This result is the same as in Compton scattering [15]. Many corrections, as for instance, those due to the proton mass cancel in (58) to a large extent [24].

Before closing this section a remark regarding the quark helicity-flip contribution is in order. Its dominance leads to very different results as we have already noted in Sect. 3.2. Thus, for instance, if we take only the leading form factor, $S_{T}$, into account (see (12)), the dominance of $C_{1}^{P}$ leads to $A_{L L}^{P}=0$ while a dominant $C_{4}^{P}$ provides $A_{L L}^{P}=1$.

At JLAB the observables $K_{L L}$ and $K_{L S}$ have recently been measured for $\pi^{0}$ photoproduction [32] in a kinematical range however in which the handbag approach cannot be applied. Measurements of these observables at higher energies are needed.

\subsection{Generalisation to other pseudoscalar mesons}

Up to this point we have concentrated on photoproduction of pions. However, nearly all that we have said holds for other pseudoscalar mesons as well and is, in fact, written in a form that allows a straightforward generalisation. Thus, the handbag amplitudes (4), (5), (11) (with the nucleon mass replaced by an appropriate one) hold directly as well as the covariant decomposition (15), (19), the subprocess observables and the twist-2 and twist-3 results. One only has to insert the relevant form factors and flavour factors $\mathcal{C}_{P}^{a b}$. For the 
process $\gamma p \rightarrow K^{+(0)} \Sigma^{0(+)}$, one has for instance

$$
R_{i, p \rightarrow \Sigma^{+}}^{d s} \simeq \sqrt{2} R_{i, p \rightarrow \Sigma^{0}}^{u s} \simeq-R_{i}^{d}+R_{i}^{s}
$$

and $\mathcal{C}_{K^{+}}^{u s}=1, \mathcal{C}_{K^{0}}^{d s}=1$. All other flavour factors are zero. The form factors (59) as well as those for other processes can easily be obtained from the information given in [16].

The results for the spin correlations discussed in Sect. 4.3, hold for other pseudoscalar mesons as well. In particular, if $C_{2}^{P}$ dominates in a given process, then (55) and (57) are approximately valid. One has to be aware that the mass of the nucleon in the definition of the quantity $\beta$ (in (5), (11), too) has to be replaced appropriately. Also, the ratio of $A_{L S}$ and $A_{L L}$ (58), holds. Ratios of cross sections, analogue to (52), can also be discussed. In this context the situation for kaons is however more intricate. Although the reaction $\gamma N \rightarrow \Sigma K$ has an isospin decomposition of the same form as in the pion photoproduction [33], the invariant functions do not have definite behaviour under $\hat{s} \leftrightarrow \hat{u}$ crossing. But if we simply generalise the leading-twist result (28) we obtain the equivalent of (37). From this we find

$$
\frac{d \sigma\left(\gamma p \rightarrow K^{0} \Sigma^{+}\right)}{d \sigma\left(\gamma p \rightarrow K^{+} \Sigma^{0}\right)}=2\left(\frac{e_{d} u+e_{s} s}{e_{u} u+e_{s} s}\right)^{2} .
$$

As for the case of pions, see (52), the form factors (59) cancel here.

When comparing photoproduction cross sections for pseudoscalar mesons not belonging to the same isospin multiplet, flavour symmetry breaking is to be considered. The decay constants play an important role in understanding many properties of flavour symmetry breaking in the pseudoscalar meson sector [34]. One may therefore expect that the decay constants also account for the main symmetry breaking effects in meson photoproduction. If so, the ratio of the $\eta$ and $\pi^{0}$ cross sections should, for instance, approximately be proportional to $\left(f_{\eta}^{\text {eff }} / f_{\pi}\right)^{2} \simeq 2$, where the effective $\eta$ decay constant may be evaluated employing the quarkflavour mixing scheme advocated for in [34]. In addition, there is a minor effect due to the different form factors in the cross section ratio. For other cross section ratios, one has to be aware of additional flavour symmetry breaking effects or, in the case of the $\eta^{\prime}$, its glue content may be of importance. As an example how to take into account the flavour mixing together with $g g$ components of the wave function we present the derivation of the leading-twist result for $\eta$ and $\eta^{\prime}$ photoproduction in the Appendix.

Finally, an interesting peculiarity is to be noted for the leading-twist calculation in the case of kaons. The kaon distribution amplitude does not exhibit the symmetry under the replacement $\tau \leftrightarrow 1-\tau$ and therefore has odd and even terms in the Gegenbauer expansion [35] in contrast to the case of the pion. As we see from (28), $C_{3}^{K}$ is related to the odd coefficients in the Gegenbauer expansion and is therefore not necessarily zero to leading-twist accuracy. According to the estimates of the lowest Gegenbauer coefficients (see, for instance, $[36]), C_{3}^{K}$ seems to amount to only about $10 \%$ of $C_{2}^{K}$ to twist-2 accuracy.

\section{Summary}

The handbag mechanism for wide-angle photoproduction of pseudoscalar mesons has been investigated. In contrast to the analysis performed in [4] where the leading-twist generation of the meson has been assumed, the partonic subprocess, $\gamma q \rightarrow P q$, has here been treated by means of the CGLN covariant decomposition [18]. The associated four invariant functions 
encode the dynamics of the subprocess and in particular that of the meson generation. This way we can consider quark helicity flip and non-flip contributions. While in the latter case the treatment of the handbag is analogue to that occurring in wide-angle Compton scattering $[4,13]$, the quark helicity-flip contribution necessitates the introduction of helicity-flip GPDs and the associated form factors about which not much is known at present.

Depending on the relative magnitudes of the invariant functions, the handbag approach leads to the characteristic predictions for ratios of cross sections and spin correlations. Provided quark helicity flip can be neglected, the data [31] on the ratio of the $\pi^{-}$and $\pi^{+}$cross sections give a strong indication at the dominance of the invariant function $C_{2}$. It would be interesting to see whether the data on the helicity correlation support this finding. If so, we would be tempted to conclude that the handbag mechanism is at work in meson photoproduction with invariant function for the subprocess which respects the relative order of magnitude, as predicted by a calculation of the twist- 2 and twist- 3 contributions, although their abso-

lute size must be larger. It remains to be seen then whether resummation of perturbative corrections or higher twist effects may lead to such a large invariant function $C_{2}$.

\section{Acknowledgements}

The authors are grateful to Andrei Belitsky, Vladimir Braun, Markus Diehl, Thorsten Feldmann, Blaženka Melić, and Wolfgang Schweiger for valuable comments. This work was partially supported by the Ministry of Science and Technology of Republic of Croatia under Contract No. 0098002 and by the ESOP network under Contract No. HPRN-CT-2000-00130.

\section{A The leading-twist result for $\eta$ and $\eta^{\prime}$ photoproduction}

From the discussions in the preceding sections it has become clear that the twist-2 contribution does not dominate wide-angle photoproduction of pseudoscalar mesons in the kinematical range currently accessible. Despite of this we will provide the leading-twist result for $\eta$ and $\eta^{\prime}$ photoproduction for the sake of completeness and for possible future use.

For the $\eta$ and $\eta^{\prime}$ mesons, mixing is to be taken into account. It is advantageous for a perturbative calculation to choose $\mathrm{SU}(3)_{F}$ singlet and octet flavour combinations of quarkantiquark states as the valence Fock states of $\eta$ and $\eta^{\prime}$ mesons [21]. The photoproduction amplitudes are then calculated for these combinations separately with corresponding distribution amplitudes and decay constants $f_{\eta}^{i}, f_{\eta^{\prime}}^{i}, i=1,8$. The numerical values of these decay constants have been evaluated in Ref. [34]:

$$
\begin{array}{ll}
f_{\eta}^{8}=1.17 f_{\pi}, & f_{\eta}^{1}=0.19 f_{\pi} \\
f_{\eta^{\prime}}^{8}=-0.46 f_{\pi}, & f_{\eta^{\prime}}^{1}=1.15 f_{\pi} .
\end{array}
$$

The photoproduction amplitudes for $\eta$ or $\eta^{\prime}$ production off protons are the sum of the corresponding singlet and octet amplitudes. In addition to the $q \bar{q}$ combinations there is also a two-gluon state which also possesses flavour-singlet quantum numbers and contributes to leading twist. For electroproduction, the corresponding amplitude for longitudinally polarised virtual photons has been calculated in [21]. Owing to the smallness of $f_{\eta}^{1}$ the two-gluon contribution to $\eta$ photoproduction is unimportant, in contrast to $\eta^{\prime}$ production where it may be sizable. 

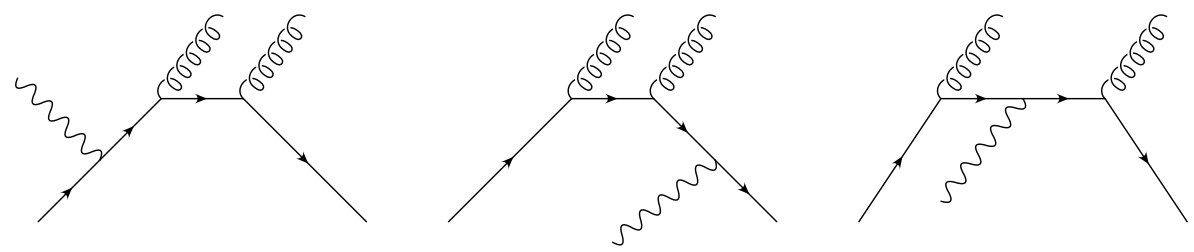

Figure 8: LO Feynman diagrams that contribute to the subprocess amplitude $\gamma q_{a} \rightarrow(g g) q_{a}$. Three more graphs are obtained by crossing the gluon lines.

Following Ref. [21], we assume that mixing is completely embedded in the decay constants and use particle-independent distribution amplitudes $\phi_{P i} \equiv \phi_{i}$, where $P=\eta, \eta^{\prime}$. As remarked above, the $P=\eta, \eta^{\prime}$ amplitudes are expressed in terms from the contributions of their flavouroctet and flavour-singlet $q \bar{q}$ Fock components as well as from the $g g$ one. The former are given by (28) with $f_{P} \rightarrow f_{P}^{i}, \phi_{P} \rightarrow \phi_{i}$, and $\mathcal{C}_{P}^{a b} \rightarrow \mathcal{C}_{i}^{a b}$, where $i=8,1$. The numerical values of the flavour factors are

$$
\begin{aligned}
& \mathcal{C}_{8}^{u u}=\mathcal{C}_{8}^{d d}=\frac{1}{\sqrt{6}}, \quad \mathcal{C}_{8}^{s s}=-\frac{2}{\sqrt{6}}, \\
& \mathcal{C}_{1}^{u u}=\mathcal{C}_{1}^{d d}=\mathcal{C}_{1}^{s s}=\frac{1}{\sqrt{3}},
\end{aligned}
$$

and $\mathcal{C}_{i}^{a b}=0$ for other flavour combinations. The amplitudes $\gamma q_{a} \rightarrow(g g) q_{a}$ can easily be computed from the Feynman diagrams displayed in Fig. 8. Using the normalisations of the two-gluon distribution amplitude and the associated one of the two-gluon twist-2 projector as proposed in Ref. [21], one finds the following result for the invariant function $C_{2}^{P}$ :

$$
\begin{aligned}
\left.C_{2}^{P(a a)}\right|_{\text {twist-2 }}= & 4 \pi \alpha_{s}\left(\mu_{R}^{2}\right) \frac{C_{F}}{N_{C}} \frac{e_{a}}{\hat{u} \hat{s}}\left\{f_{P}^{8} \mathcal{C}_{8}^{a a}\langle 1 / \tau\rangle_{8}\right. \\
& \left.+f_{P}^{1} \mathcal{C}_{1}^{a a}\langle 1 / \tau\rangle_{1}-f_{P}^{1} \frac{1}{\sqrt{n_{f}}}\left\langle 1 / \tau^{2}\right\rangle_{g}\right\}, \\
\left.C_{1}^{P(a a)}\right|_{\text {twist-2 }}= & \left.C_{3}^{P(a a)}\right|_{\text {twist-2 }}=\left.C_{4}^{P(a a)}\right|_{\text {twist-2 }}=0,
\end{aligned}
$$

with $n_{f}=3$ being the numbers of quark flavours. The invariant function $C_{3}^{P(a a)}$ is zero to leading-twist accuracy since the distribution amplitudes $\phi_{8,1}$ are symmetric under the replacement $\tau \leftrightarrow 1-\tau$ and the gluon contribution is zero even at parton level. The moment of the two-gluon distribution amplitude, which is antisymmetric under the replacement $\tau \leftrightarrow 1-\tau$ and which mixes under evolution with $\phi_{1}$, is defined by

$$
\left\langle 1 / \tau^{2}\right\rangle_{g}=\int_{0}^{1} d \tau \frac{\phi_{g}(\tau)}{\tau^{2}} .
$$

In [21], the twist-2 gluon distribution $\phi_{g}$ amplitude has been estimated from a NLO analysis of the $\gamma \rightarrow \eta, \eta^{\prime}$ transition form factors. The Gegenbauer series of the distribution amplitudes have been truncated at $n=2$; for the only non-zero Gegenbauer coefficient, a value of $B_{2}^{g} \equiv B_{2}^{g}\left(\mu_{0}^{2}\right)=9 \pm 12$ has been found at a scale of $\mu_{0}^{2}=1 \mathrm{GeV}^{2}$. This coefficient is connected, through evolution, with the Gegenbauer coefficient $B_{2}^{1} \equiv B_{2}^{1}\left(\mu_{0}^{2}\right)=-0.08 \pm 0.04$ appearing in the expansion of $\phi_{1}$. It is to be stressed that the evolutional effects play an important role for the distribution amplitudes that differ from the asymptotic form $\left(\phi_{1}=\right.$ 
$\left.\phi_{8}=6 \tau(1-\tau), \phi_{g}=0\right)$. Recently, a combined analysis of the transition form factors and the inclusive $\Upsilon(1 S) \rightarrow \eta^{\prime} X$ decay lead to more severe restrictions of the Gegenbauer coefficients [37], namely, $B_{2}^{(g)} \approx 8 \pm 5$ and $B_{2}^{1} \approx-0.07 \pm 0.03$ at $\mu_{0}^{2}=1 \mathrm{GeV}^{2}$. In any case, the twogluon contribution may be sizable and could enhance the cross section for $\eta^{\prime}$ photoproduction substantially.

\section{References}

[1] D. Müller, D. Robaschik, B. Geyer, F. M. Dittes and J. Hořejši, Fortsch. Phys. 42, 101 (1994) [hep-ph/9812448]; A.V. Radyushkin, Phys. Rev. D56, 5524 (1997) [hepph/9704207]; X. Ji, Phys. Rev. D55 7114 (1997) [hep-ph/9609381].

[2] A.V. Radyushkin, Phys. Lett. B385, 333 (1996) [hep-ph/9605431]; J.C. Collins, L. Frankfurt and M. Strikman, Phys. Rev. D56, 2982 (1997) [hep-ph/9611433].

[3] L. Mankiewicz and G. Piller, Phys. Rev. D61, 074013 (2000) [hep-ph/9905287].

[4] H. W. Huang and P. Kroll, Eur. Phys. J. C17, 423 (2000), [hep-ph/0005318].

[5] L. Mankiewicz, G. Piller and T. Weigl, Eur. Phys. J. C5, 119 (1998) [hep-ph/9711227]; M. Vanderhaeghen, P. A. Guichon and M. Guidal, Phys. Rev. D60, 094017 (1999) [hep$\mathrm{ph} / 9905372]$.

[6] G. P. Lepage and S. J. Brodsky, Phys. Rev. D22, 2157 (1980). A. V. Efremov and A. V. Radyushkin, Phys. Lett. B94, 245 (1980).

[7] R. Jakob and P. Kroll, Phys. Lett. B315, 463 (1993) [Erratum-ibid. B319, 545 (1993)] [hep-ph/9306259].

[8] S. J. Brodsky, C. R. Ji, A. Pang and D. G. Robertson, Phys. Rev. D57, 245 (1998) [hep-ph/9705221]; B. Melić, B. Nižić and K. Passek, Phys. Rev. D60, 074004 (1999) [hep-ph/9802204].

[9] C. J. Bebek et al., Phys. Rev. D17, 1693 (1978); J. Volmer et al. [The Jefferson Lab F(pi) Collaboration], Phys. Rev. Lett. 86, 1713 (2001) [nucl-ex/0010009].

[10] P. Eden, P. Hoyer and A. Khodjamirian, JHEP 0110 (2001) 040 [arXiv:hep-ph/0110297].

[11] A. Belitsky, hep-ph/0307256.

[12] A. V. Radyushkin, Phys. Rev. D58, 114008 (1998) [hep-ph/9803316].

[13] M. Diehl, T. Feldmann, R. Jakob and P. Kroll, Eur. Phys. J. C8, 409 (1999) [hep$\mathrm{ph} / 9811253]$.

[14] M. Diehl, Eur. Phys. J. C19, 485 (2001) [hep-ph/0101335].

[15] H. W. Huang, P. Kroll and T. Morii, Eur. Phys. J. C23, 301 (2002) [hep-ph/0110208].

[16] L. L. Frankfurt, P. V. Pobylitsa, M. V. Polyakov and M. Strikman, Phys. Rev. D60, 014010 (1999) [hep-ph/9901429]. 
[17] P. Hoodbhoy and X. Ji, Phys. Rev. D58, 054006 (1998) [hep-ph/9801369].

[18] G. Chew, M. Goldberger, F. Low and Y. Nambu, Phys. Rev. 106, 1345 (1957).

[19] M. Diehl, T. Feldmann, R. Jakob and P. Kroll, Phys. Lett. B460, 204 (1999) [hep$\mathrm{ph} / 9903268]$.

[20] M. Beneke and T. Feldmann, Nucl. Phys. B592, 3 (2001) [hep-ph/0008255].

[21] P. Kroll and K. Passek-Kumerički, Phys. Rev. D 67, 054017 (2003) [hep-ph/0210045].

[22] V. M. Braun and I. E. Halperin, Z. Phys. C48, 239 (1990) [Sov. J. Nucl. Phys. 52, 126 (1990 YAFIA,52,199-213.1990)].

[23] P. Ball, JHEP 9901, 010 (1999) [hep-ph/9812375].

[24] M. Diehl, T. Feldmann, R. Jakob and P. Kroll, Nucl. Phys. B596, 33 (2001) [Erratumibid. B605, 647 (2001)] [hep-ph/0009255].

[25] M. Glück, E. Reya and A. Vogt, Eur. Phys. J. C5, 461 (1998) [hep-ph/9806404]; A. D. Martin, R. G. Roberts, W. J. Stirling and R. S. Thorne, Eur. Phys. J. C23, 73 (2002) [hep-ph/0110215].

[26] P. Kroll, hep-ph/0302169.

[27] O. Gayou et al. [Jefferson Lab Hall A Collaboration], Phys. Rev. Lett. 88, 092301 (2002) [nucl-ex/0111010].

[28] J. Arrington, nucl-ex/0305009; L. Andivahis et al., Phys. Rev. D 50, 5491 (1994).

[29] P. G. Blunden, W. Melnitchouk and J. A. Tjon, nucl-th/0306076; P. A. Guichon and M. Vanderhaeghen, hep-ph/0306007.

[30] M. Diehl, T. Feldmann, H. W. Huang and P. Kroll, Phys. Rev. D67, 037502 (2003) [hep-ph/0212138].

[31] L. Y. Zhu et al. [Jefferson Lab Hall A Collaboration], nucl-ex/0211009.

[32] K. Wijesooriya et al. [Jefferson Lab Hall A Collaboration], Phys. Rev. C 66 (2002) 034614 .

[33] A. Donnachie, in: High Energy Physics, Vol V., edited by E. H. S. Burhop (Academic Press, New York, London 1972), p. 1-185.

[34] T. Feldmann, P. Kroll and B. Stech, Phys. Rev. D 58, 114006 (1998) [hep-ph/9802409].

[35] V. L. Chernyak and A. R. Zhitnitsky, Phys. Rept. 112, 173 (1984).

[36] J. Bolz, P. Kroll and G. A. Schuler, Eur. Phys. J. C 2, 705 (1998) [hep-ph/9704378]; T. Shigetani, K. Suzuki and H. Toki, Phys. Lett. B 308, 383 (1993) [hep-ph/9402286];

A. E. Dorokhov, Nuovo Cim. A 109, 391 (1996).

[37] A. Ali and A. Y. Parkhomenko, hep-ph/0304278. 